\title{
Cathodoluminescence Study of Microdiamonds and Improvements of Signal Detection by Lowering Temperature of the Sample
}

\author{
Nadezda Vaskovicova ${ }^{1}$, Radim Skoupy $^{1}$, Ales Patak ${ }^{1}$, Kamila Hrubanova ${ }^{1}$ and Vladislav Krzyzanek ${ }^{1}$ \\ ${ }^{1 .}$ Institute of Scientific Instruments of the CAS, v. v. i., Brno, Czech Republic
}

Cathodoluminescence (CL) is one of the optical properties all minerals have; the special part of research related belongs to natural and synthetic diamonds. CL can be also used to distinguish between natural and synthetic diamonds [1,2,3]. Moreover, CL can be used to examine impurities, dopants and defects in diamonds [2]. In this study, the synthetic microdiamonds were used to determine the dependence of CL spectrum in the scanning electron microscope (SEM) on sample temperature ranging from room temperature to cryogenic temperatures.

The gold carrier was moistened with ethanol, then a small amount of diamond particles was dusted on the surface and dried in the ACE600 device (Leica microsystems) at high vacuum $\left(10^{-5} \mathrm{~Pa}\right)$ and at room temperature. In the high vacuum, the sample was transferred to the SEM Magellan 400 (FEI) using the shuttle VCT100 (Leica microsystem), and placed into the cryo-holder (Leica microsystems), still retaining the room temperature. To reduce sample contamination during the imaging, it was being cleaned with the nitrogen plasma for 2 minutes. Separated microdiamonds were localized and selected for CL analysis (Figure 1) using the detection system MonoCL4 (Gatan). The SEM conditions for CL spectra acquisition were: electron energy of $3 \mathrm{keV}$, electron probe current of $13 \mathrm{pA}$, working distance of $7.3 \mathrm{~mm}$, magnification of 8000; the CL spectrum was recorded in the wavelength range from $200 \mathrm{~nm}$ to $970 \mathrm{~nm}$, the photomultiplier tube detector voltage $1200 \mathrm{~V}$ and the acquisition time of one spectrum 12 minutes 50 seconds.

The CL measurements started at room temperature $\left(+26^{\circ} \mathrm{C}\right)$, the SEM specimen chamber pressure was $10^{-4} \mathrm{~Pa}$. In the course of the following steps the temperature of the cryo-holder gradually decreased and the CL spectra were recorded at the temperatures: $-80{ }^{\circ} \mathrm{C},-100{ }^{\circ} \mathrm{C},-120{ }^{\circ} \mathrm{C}$ and $-149{ }^{\circ} \mathrm{C}$. All $\mathrm{CL}$ spectra are combined in Figure 2 that shows the decreasing noise and increasing signal from $+26^{\circ} \mathrm{C}$ to $-100{ }^{\circ} \mathrm{C}$; the number of CL counts at the main peak is about $30 \%$ higher at $-100{ }^{\circ} \mathrm{C}$ in comparison to $+26{ }^{\circ} \mathrm{C}$.

At temperatures of $-120^{\circ} \mathrm{C}$ and $-149^{\circ} \mathrm{C}$ the $\mathrm{CL}$ signal decreased; thus, the number of CL counts at the main peak is at $-149{ }^{\circ} \mathrm{C}$ only about $44 \%$ of the one at $-100{ }^{\circ} \mathrm{C}$. We assume that the contamination on the diamonds surface (Figure 3A) can partially block the CL signal. At the end of the experiment the diamonds were transferred back to the ACE600 device and dried at high vacuum $\left(10^{-5} \mathrm{~Pa}\right)$, and they were observed the next day in the SEM for a contamination residuum (Figure 3B). The diamonds seem to be clean in these images. We suppose that the source of contamination is water ice or nitrogen.

This leads us to a conclusion that the proximity of CL collector mirror, long distance and small area of original cryo-decontamination shield are sufficiently effective for the cryo-CL measurements in the SEM Magellan only down to the temperature of $-100{ }^{\circ} \mathrm{C}$. For lower temperatures we have to investigate new design of a cryo-holder and anti-contaminator elements which can improve and extend the possibilities for measurement in this device [4].

References: 
[1] BG Yacobi, DB Holt, Cathodolumin. microscopy of inorganic solids, (Springer, New York) p. 262.

[2] H Kawarada et al, J. Appl. Phys. 67 (1990), p. 983.

[3] YL Khong and AT Collins, Diamond and Related Materials 2 (1993), p. 1.

[4] The authors acknowledge the support from the Grant Agency of the Czech Republic (GA1715451S) and Ministry of Education, Youth and Sports of the Czech Republic (LO1212) together with the European Commission (ALISI No. CZ.1.05/2.1.00/01.0017).
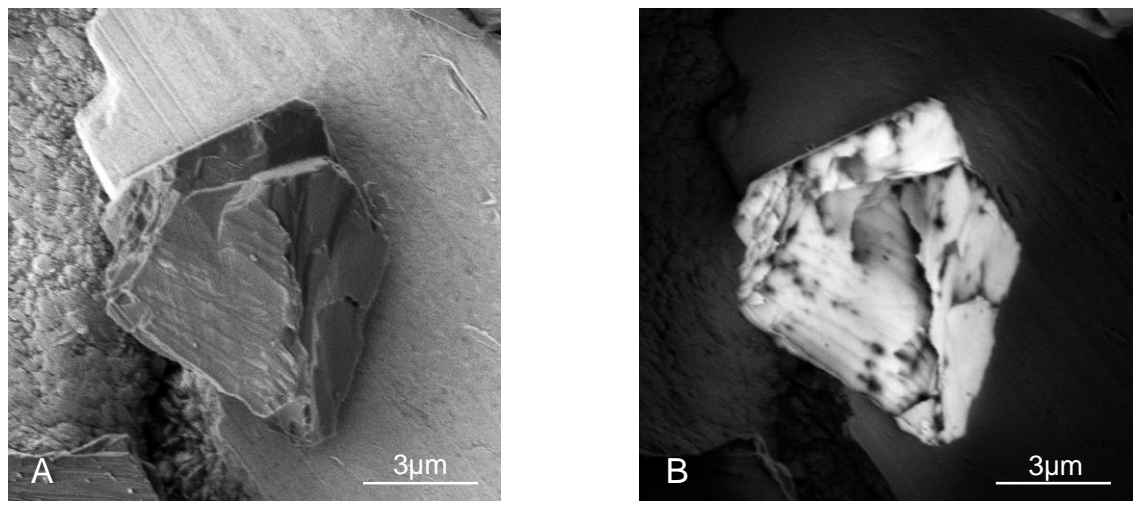

Figure 1. A. SEM image and $\mathbf{B}$ the same view acquired in the CL mode of a microdiamond.

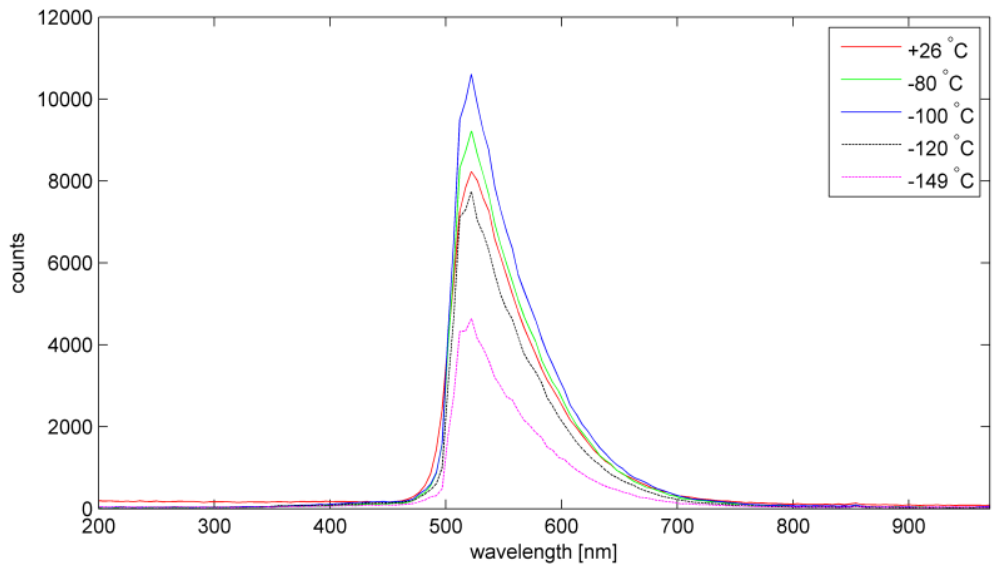

Figure 2. The CL-spectra of the same microdiamond recorded at different temperatures going from higher to lower.
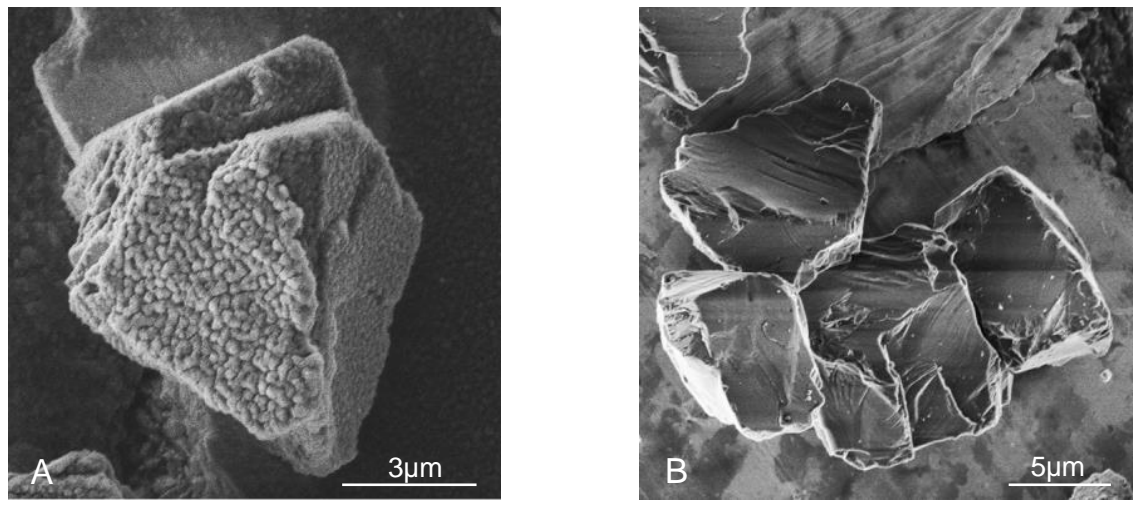

Figure 3. A. Cryo-SEM image of a microdiamond at the temperature of $-149^{\circ} \mathrm{C}$ showing high contamination. B. SEM image of microdiamonds after vacuum drying recorded at room temperature. 